\title{
On Inner $\rho$-Derivations
}

Dedicated to Professor Yoshie Katsurada on the occasion of her 60th birthday

\section{By Kazuo Kishimoto and Hisao Tominaga}

Throughout the present paper, $A$ will represent a ring with $1, C$ the center of $A$, and $B$ a subring of $A$ containing 1 . We consider a (ring) endomorphism $\rho$ of $A$ sending 1 to 1 , and set $J(\rho)=\{x \in A \mid \boldsymbol{\rho}(x)=x\}$. Further, we use the following convention : $a, a^{\prime}$ will mean arbitrary elements of $A$, and $b, b^{\prime}$ elements of $B$.

Given $x \in A$, the map $\delta_{\rho, x}: A \rightarrow A$ defined by $\delta_{\rho, x}(a)=a x-x \rho(a)$ is

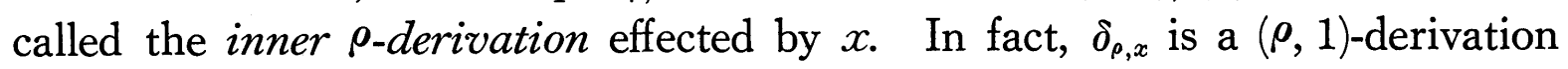
of $A: \delta_{\rho, x}\left(a a^{\prime}\right)=\delta_{\rho, x}(a) \rho\left(a^{\prime}\right)+a \delta_{\rho, x}\left(a^{\prime}\right)$. Recently, in his paper [3], P. V. Praag proved the following proposition: If $A$ is a division ring with $[A: C]>4$ and $B$ is a division subring properly contained in $A$ which is invariant relative to all the inner $\rho$-derivations effected by elements of $A$, then $B$ is a field contained in $J(\rho)$.

In what follows, we shall prove a proposition which essentially contains Praag's, and extend [3; Corollaire] to two-sided simple rings and (right) primitive rings with non-zero socles.

Lemma 1. Assume that $B$ is invariant relative to all the inner $\rho_{-}$ derivations effected by elements of $A$.

(a) The restriction $\rho \mid B$ of $\rho$ to $B$ is an endomorphism of $B$.

(b) If $B$ is commutative then the additive group $[B, A]$ generated by $\{[b, a]=a b-b a \mid a \in A, b \in B\}$ is a $B$-B-submodule of $A$ and $b d=d \rho(b)$ for every $d \in[B, A]$.

PRoof. (a) is evident by $b-\boldsymbol{\rho}(b)=b \cdot 1-1 \cdot \boldsymbol{\rho}(b) \in B$. If $B$ is commutative, it is easy to see that $[B, A]$ is $B$ - $B$-admissible. Further, $\left[b, b^{\prime} a-a \rho\left(b^{\prime}\right)\right]=0$ implies $b^{\prime}[b, a]=[b, a] \rho\left(b^{\prime}\right)$, proving (b).

Lemma 2. Let $B$ be a field. Assume that $B$ is invariant relative to all the inner $\rho$-derivations effected by elements of $A$ and $B \nsubseteq J(\rho)$.

(a) $B$ coincides with the centeralizer $V_{A}(B)$ of $B$ in $A$.

(b) $A=[B, A]+B$ and $[B, A]^{2} \subseteq B$.

Proof. (a) It suffices to prove $V_{A}(B) \subseteq B$. Now, let $b^{*}$ be an arbitrary element of $B$ not contained in $J(\rho)$. If $v$ is in $V_{A}(B)$ then $v\left(b^{*}-\rho\left(b^{*}\right)\right)=$ 
$b^{*} v-v \boldsymbol{\rho}\left(b^{*}\right) \in B$ and $b^{*}-\rho\left(b^{*}\right)$ is a non-zero element of $B$ (Lemma 1 (a)). Hence, $v$ is in $B$.

(b) There holds $a=\left(\rho\left(b^{*}\right)-b^{*}\right)^{-1}\left(\left[\rho\left(b^{*}\right), a\right]-\left(b^{*} a-a \rho\left(b^{*}\right)\right) \in[B, A]+B\right.$ by Lemma 1 (b). Further, if $d, d^{\prime}$ are in $[B, A]$ then $\left(d d^{\prime}\right)\left(\rho^{2}\left(b^{*}\right)-\rho\left(b^{*}\right)\right)=b^{*}$ $\left(d d^{\prime}\right)-\left(d d^{\prime}\right) \rho\left(b^{*}\right) \in B\left(\right.$ Lemma 1 (b)). Since $\rho^{2}\left(b^{*}\right)-\boldsymbol{\rho}\left(b^{*}\right) \neq 0$, we obtain $d d^{\prime} \in B$.

Proposition 1. Assume that $B$ is a proper subfield of $A$ which is invariant relative to all the inner $\rho$-derivations effected by elements of $A$. If $B \nsubseteq J(\rho)$ and $A$ is a prime ring then $A$ is a simple ring with $[A: C]=4$.

Proof. Since $A=[B, A]+B$ by Lemma 2 (b), $[B, A]^{2} \neq 0$. In fact, $[B, A]^{2}=0$ implies a contradition that $[B, A]$ is a non-zero nlipotent ideal of $A$. Now, let $u, u^{\prime}$ be elements of $[B, A]$ with $u u^{\prime} \neq 0$. Then, $u u^{\prime}$ is a unit of $B$ by Lemma 2 (b) and $u^{\prime} u$ is also in $B$ and non-zero by $\left(u u^{\prime}\right)^{2} \neq 0$. We see therefore that $u$ is a unit. Since $b u=u \rho(b)$ by Lemma 1 (b), we obtain $b\left(\left[b^{\prime}, a\right] u^{-1}\right)=\left[b^{\prime}, a\right] \rho(b) u^{-1}=\left[b^{\prime}, a\right]\left(u^{-1} b u\right) u^{-1}=\left(\left[b^{\prime}, a\right] u^{-1}\right) b$. Hence, $\left[b^{\prime}, a\right] u^{-1} \in V_{A}(B)=B($ Lemma 2 $(\mathrm{a}))$, namely, $[B, A]=B u$. We have seen therefore $[A: B]_{L}=2$. Combining this with $B=V_{A}(B)$, we readily obtain $[A: C]=4$. (Cf. for instance [5; Proposition 7.1].)

Lemma 3. Assume that $B$ is a proper subring of $A$ which is invariant relative to all the inner $\rho$-derivations effected by elements of $A$.

(a) If $B$ is two-sided simple then $B$ is a field.

(b) If $A$ is a (right) primitive ring with non-zero socle $S, B$ a completely primitive ring (with non-zero socle $T$ ), and $\rho$ a monomorphism, then $B$ is a field.

Proof. (a) Since $b\left(b^{\prime} a-a \rho(b)\right) \in B$ and $b^{\prime}(b a)-(b a) \rho\left(b^{\prime}\right) \in B$, we readily obtain $\left[b, b^{\prime}\right] a \in B$. If $[B, B] \neq 0$ then $B[B, B] B=B$, and hence $A=B A=$ $(B[B, B] B) A \subseteq B$. This contradiction shows that $B$ is a field.

(b) By [1; Theorem IV. 15.1], $T$ can not be equal to $S$. As in (a), we obtain $B \supseteq[B, B] A \cup A \rho([B, B])$. If $[B, B] \neq 0$ then $\rho([B, B] \neq 0$. Noting here that the non-zero ideals $B[B, B] B$ and $B \boldsymbol{\rho}([B, B]) B$ contains $T$, we readily obtain $B \supseteq T A$ and $B \supseteq A T$. Accordingly, $T \subseteq T A=T T A \subseteq T$ and $T \subseteq A T \subseteq A T T \subseteq T$, namely, $A T=T=T A$. Hence, the ideal $T$ of $A$ contains $S$ and $S T$ is a non-zero ideal of $B$, which implies a contradiction $S=T$.

LeMmA 4. If $A$ is a simple ring with $[A: C]=4$ and $B$ is a field with $B=V_{A}(B)$, then there exists a unique automorphism $\rho_{B}$ of $B$ such that $b a-a \rho_{B}(b) \in B$, and then there exists an (inner) automorphism $\rho^{\prime}$ of $A$ such that $B$ is invariant relative to all the inner $\rho^{\prime}$-derivations effected by elements of $A$. 
Proof. Let $x$ be an arbitrary element of $A$ not contained in $B$. Then, $x, 1$ form a right free $B$-basis of $A$, and we obtain a map $\rho_{B}: B \rightarrow B$ such that $\left(b x-x \boldsymbol{\rho}_{B}(b) \in B\right.$ and hence) $b a-a \rho_{B}(b) \in B$. To be easily seen, $\rho_{B}$ is a $C$-automorphism of $B$ and uniquely determined. Now, it is well-known that $\rho_{B}$ can be extended to an inner automorphism $\rho^{\prime}$ of $A$. (Cf. for instance [5; Theorem 7.2].)

Theorem 1. Let $A$ and $B$ be two-sided simple. Then, $B$ is invariant relative to all the inner $\rho$-derivations effected by elements of $A$ if and only if one of the following conditions is satisfied:

(1) $B=A$.

(2) $B \subseteq J(\rho) \cap C$.

(3) $[A: C]=4, B=V_{A}(B)$, and $\rho \mid B=\rho_{B}$ (see Lemma 4).

THEOREM 2. Let $A$ be a primitive ring with non-zero socle, $B$ a completely primitive ring, and $\rho$ a monomorphism. Then, $B$ is invariant relative to all the inner $\rho$-derivations effected by elements of $A$ if and only if one of the following conditions is satisfied:

(1) $B=A$.

(2) $B \subseteq J(\boldsymbol{\rho}) \cap C$.

(3) $A$ is a simple ring with $[A: C]=4, B=V_{A}(B)$, and $\rho \mid B=\rho_{B}$.

Proof of Theorems 1 and 2. By the validity of Lemma 4 , it remains only to prove the only if part. We may assume here that $B$ is a proper subring of $A$. In any rate, $B$ is a field by Lemma 3. If $B \not J(\boldsymbol{\rho})$ then it is the case (3) by Proposition 1 and Lemma 4. On the other hand, if $B \subseteq J(\rho)$ then $B$ is invariant relative to all the inner derivations effected by elements of $A$. In case $B$ is not of characteristic $2, B \subseteq C$ by [5; Proposition $8.10(\mathrm{~b})]$ and $\left[2 ;\right.$ Corollary $\left.1\left(1^{\prime}\right)\right]$. In what follows, we may assume therefore that $B$ is of characteristic 2 and $B \nsubseteq C$. Then, $[b,[b, a]]=0$ implies $b^{2} \in C$, in particular, $[b, a]^{2} \in C$. Hence, $A$ is a simple ring with $[A: C]=4$ by [4; Theorem 2]. Choosing here $b_{0} \in B$ and $a_{0} \in A$ with $\left[b_{0}, a_{0}\right] \neq 0$, there holds $1=\left[b_{0}, a_{0}\right]^{-1}\left[b_{0}, a_{0}\right]=\left[b_{0},\left[b_{0}, a_{0}\right]^{-1} a_{0}\right]$. Accordingly, for every $c \in C$ we have $c=\left[b_{0}, c\left[b_{0}, a_{0}\right]^{-1} a_{0}\right] \in B$, which means $C \varsubsetneqq B$. Consequently, $B=V_{A}(B)$ and it is the case (3).

\author{
Departments of Mathematics, \\ Shinshu University \\ Okayama University
}

\title{
References
}

[1] N. Jacobson: Structure of Rings, Amer. Math. Soc. Colloq. Publ., 37, 1956. 
[2] K. Motose : On Cartan-Brauer-Hua theorem, J. Fac. Sci. Hokkaido Univ., Ser. I, 20 (1967), 27-30.

[3] P. V. PRAAG: S-dérivations internes dans les corps, J. of Algebra, 17 (1971), 470-473.

[4] M. SlATER: On simple rings satisfying a type of "restricted" polynomial identity, J. of Algebra, 1 (1964), 347-354; Erratum, 2 (1965), 152.

[5] H. Tominaga and T. NAgahara: Galois Theory of Simple Rings, Okayama Math. Lectures, 1970.

(Received November 29, 1971) 